\title{
EL CENSO COMO PRODUCTOR DE ADJUDICATARIOS EN LAS POLÍTICAS HABITACIONALES.
}

\section{THE CENSUS AS A PRODUCER OF SUCESSFUL TENDERERS IN HOUSING POLICIES.}

\author{
Romina Olejarczyk ${ }^{1}$
}

\section{Resumen}

En este artículo me propongo reflexionar acerca de los instrumentos de recolección de datos denominados censos o verificaciones- frecuentemente utilizados en el marco de los programas de construcción de viviendas, a los fines de establecer a quienes se adjudicarán. En particular, me abocaré en la aplicación de una política pública que se lleva a cabo en la Argentina desde el año 2003, conocida como los Programas Federales de Construcción de viviendas, en un municipio del Área Metropolitana de Buenos Aires. El análisis de la experiencia de aplicación del censo, para el caso de este municipio, me permitirá conocer qué dilemas y conflictos se presentan al momento de su utilización, así como reflexionar acerca de los sentidos que los sujetos implicados le atribuyen a estos procesos. La información que aquí mencionaré surge de un trabajo de campo etnográfico, llevado a cabo durante los años 2008-2012.

Palabras claves: programas de construcción de viviendas, instrumentos de recolección de datos, sujetos implicados.

\begin{abstract}
In this article, I intend to reflect on the data collection instruments - censuses or verificationsfrequently used in the context of housing construction programs, in order to establish who will be awarded. In particular, I will deal in implementing public policy that is carried out in Argentina since 2003, known as the Federal Programs Housing construction in a municipality of Greater Buenos Aires. The analysis of implementation experience census, in the case of this town, let me know what dilemmas and conflicts arise at the time of use, and reflect on the meanings attributed the subjects involved in these processes. The information I will mention here arises from an ethnographic fieldwork, conducted during the years 2008-2012.
\end{abstract}

Keywords: housing construction programs, data collection instruments, subjects involved.

\footnotetext{
${ }^{1}$ Investigadora- Instituto de Investigaciones Gino Germani, Facultad de Ciencias Sociales, Universidad de Buenos Aires y Consejo Nacional de Investigaciones Científicas y Tecnológicas (CONICET). E-mail: romi_olejar@yahoo.com.ar
} 


\section{INTRODUCCIÓN}

En este artículo me propongo reflexionar acerca de los instrumentos de recolección de datos -denominados censos o verificaciones- frecuentemente utilizados en el marco de los programas de construcción de viviendas, a los fines de establecer a quienes se adjudicarán las viviendas construidas.

En particular, me abocaré en la aplicación de una política pública que se lleva a cabo en la Argentina desde el año 2003, conocida como los Programas Federales de Construcción de viviendas ${ }^{1}$, en un municipio del Área Metropolitana de Buenos Aires².

El análisis de la experiencia de aplicación del censo para el caso de este municipio, me permitirá conocer qué dilemas y conflictos se presentan al momento de su utilización, así como aproximarme a los sentidos que los sujetos implicados le atribuyen a estos procesos.

La información que aquí mencionaré surge de un trabajo de campo etnográfico llevado a cabo durante los años 2008-2012. Durante este período, además de la observación participante, llevé a cabo entrevistas abiertas con habitantes de unos de los barrios construidos, profesionales que participaron en el proceso de adjudicación y funcionarios a cargo de la aplicación de los Programas Federales a escala del gobierno local, provincial y nacional³.

\section{BREVE RESEÑA DE LOS PROGRAMAS FEDERALES}

Los Programas Federales forman parte de la política habitacional que el gobierno argentino lleva a cabo desde el año 2003. Estos programas fueron promovidos con los objetivos de: generar empleo, impactar en la economía local -a partir de la movilización del mercado de la construcción- y la disminución del déficit habitacional. La estrategia política para cumplir con los mismos ha sido la inversión en obra pública: obras de infraestructura como la ampliación de la

\footnotetext{
2 El AMBA "es la continuidad urbana de la CABA (de aproximadamente $200 \mathrm{~km} 2$ y 3.000 .000 de habitantes) y los Partidos de la Provincia de Buenos Aires que lo rodean (de aproximadamente $3.600 \mathrm{Km} 2$ y una población cercana a los 9.000.000 de habitantes)" en www.atlasbuenosaires.gov.ar a Junio 2014.

${ }^{3}$ Una primera versión de las reflexiones que forman parte de este artículo surgen de mi tesis de maestría: "Tensiones en las políticas habitacionales: el caso de los Programas Federales de Construcción de Viviendas en Avellaneda". Asimismo, he presentado una versión preliminar en las VII Jornadas Santiago Wallace de investigación en Antropología Social, organizadas por la Facultad de Filosofía y Letras de la Universidad de Buenos Aires, en Diciembre de 2013. Todos estos materiales, incluido este artículo, han sido producidos en el marco de mi Beca doctoral Tipo II financiada por el Consejo Nacional de Investigaciones científicas y Técnicas (CONICET), y de $\mathrm{mi}$ inserción en el proyecto de investigación: "Naturaleza, cultura y segregación: una lectura antropológica sobre políticas socio-urbanas hacia sectores populares en el Área Metropolitana de Buenos Aires." (UBACYT 2012-2014. Instituto de Investigaciones Gino Germani. Facultad de Ciencias Sociales de la Universidad de Buenos Aires), dirigido por la Dra. María Carman, y que forma parte de la Red de equipos de investigación CONTESTED_CITIES, recibiendo financiamiento de la línea PEOPLE-IRSES del Séptimo Programa Marco de la Comisión Europea (Contrato PIRSES-GA-2012-318944).
} 
red de agua y cloaca, asfalto y mejoramiento de calles, construcción de equipamientos urbanos - centros de salud, escuelas- y la construcción de viviendas, entre otras. Es por ello que los Programas Federales dependen de la Subsecretaría de Desarrollo Urbano y Vivienda dependiente de la Secretaría de Obras Públicas del Ministerio Planificación, Inversión Pública y Servicios de la Nación Argentina.

Dentro del cúmulo de programas que forman parte de los Programas Federales, en julio de 2004 el Gobierno Nacional anunció el lanzamiento del denominado Plan Federal de Construcción de Viviendas. Este programa, promovía la construcción de 120.000 viviendas en el período 2004-2006 en todo el territorio de la Argentina, destinándose 40.000 al AMBA. El eje puesto en la generación de empleo y la movilización del mercado de la construcción, puede identificarse claramente en los objetivos planteados en su presentación oficial:

Consolidar la evolución decreciente del déficit habitacional; fortalecer el proceso de reactivación económica del sector habitacional y de la industria de la construcción; contribuir a la disminución del índice de desempleo a través de la generación de 360.000 puestos de trabajo; instrumentar programas de capacitación para la provisión de los recursos humanos necesarios; mejorar la calidad de vida de los hogares.

Se estipuló una partida presupuestaria, monto y características de la construcción de las viviendas por región, así como cupos para cada una de ellas. Cabe señalar que, a excepción del Programa de Emergencia Habitacional que implicaba la conformación de cooperativas para la construcción de viviendas, el resto de los programas implicaba la participación de empresas privadas.

Cada uno de estos programas se desagregó en Subprogramas. A los fines de este trabajo, me interesa mencionar en particular el Subprograma de Urbanización de Villas y Asentamientos Precarios, dado que el mismo establece una diferencia con el resto de los programas, en tanto se enfoca específicamente en los habitantes de villas y asentamientos, pero además, porque incorpora la realización de obras de infraestructura, mejoramiento habitacional y barrial. Los objetivos -hasta la actualidad- son los siguientes:

Mejorar la calidad de vida de la población asentada en villas y asentamientos precarios con carencia de infraestructura básica de servicios, problemas ambientales y de regularización dominial; consolidar a la población en el sitio que habita, regularizando el dominio de la tierra a favor de sus ocupantes efectivos, proveyendo infraestructura básica de servicios, consolidando los espacios públicos, mitigando los problemas ambientales e incorporando infraestructura privada de saneamiento básico y relocalizar aquellas familias que se encuentran ubicadas en sectores urbanos de alto riesgo ambiental. 
Este Subprograma contiene una mirada que supera de algún modo el énfasis en lo constructivo, al mencionar problemáticas referidas a las características del ambiente donde se emplazarán las viviendas futuras, así como la posibilidad de regularizar dominialmente las tierras y del equipamiento urbano que utilizarán las familias. En sus comienzos, este Subprograma se concentró en el Área Metropolitana de Buenos Aires.

En abril del año 2009 el gobierno nacional anunció la continuidad de los Programas Federales. La operatoria de construcción de viviendas comenzó a denominarse "Techo Digno". De acuerdo a la información publicada, los objetivos de este Programa son: "Fortalecer el proceso de reactivación económica del sector habitacional; Consolidar la evolución decreciente del déficit habitacional registrada en la última década; Mejorar la calidad de vida de los sectores más vulnerables; Brindar al sector de la construcción un marco de previsibilidad que mejore las condiciones del mercado."

Dentro de sus objetivos se continúa mencionando el de la reactivación económica, así como el objetivo de incidir en el mercado de la construcción, esta vez generando condiciones de producción favorables para el mismo. En último lugar, se sostiene el objetivo de incidir en las condiciones de vida de la población afectada. Existe cierto corrimiento de la cuestión de generar empleo, dado que este objetivo perdió jerarquía en comparación con aquellos del primer lanzamiento de estos programas, en el marco post crisis 2001. En palabras de un entrevistado:

Al principio la cuestión era dar trabajo, o sea, poner en marcha el engranaje para que se generara trabajo. La prioridad no era tanto pensar en el déficit habitacional y diría más, tampoco mucho en la calidad de lo que se hacía porque lo importante era dar trabajo. A dos años de eso empezamos a mirar, o sea, nos pusimos un poquito más exigentes desde dos puntos de vista: desde la calidad constructiva y también desde la calidad institucional. (Entrevista al Coordinador del Programa de Urbanización de Villas y Asentamientos Precarios a escala nacional. Noviembre 2012).

Dentro de la reestructuración de los Programas Federales bajo la órbita del Techo Digno, se re categorizó el Subprograma de Urbanización de Villas y Asentamientos Precarios como Programa. Esto implicó, en principio, mayores recursos y autonomía para las intervenciones en villas y asentamientos urbanos. El primer impacto tendría que ver con que el Programa se amplió de su aplicación en el Área Metropolitana de Buenos Aires hacia el territorio de todo el país, sin embargo ésta sigue siendo el área de mayor incidencia. 
Ahora bien, este trabajo se asienta en el análisis de la experiencia de implementación de estos programas en un municipio de la zona sur del Área Metropolitana de Buenos Aires, denominado Avellaneda ${ }^{4}$.

Los Programas Federales comenzaron a implementarse allí como parte de un Plan de Urbanización de una villa conocida como Villa Tranquila. Y esta fue la primera experiencia a la cual se sumaron otras, a medida que se conseguían nuevos terrenos y más financiamiento para la construcción de viviendas ${ }^{5}$.

Asimismo, los Programas Federales se implementan desde sus comienzos -y hasta el día de hoy- siguiendo la lógica de implementación a nivel nacional a través de la Secretaría de Obras Públicas municipal. Los equipos de la Secretaría de Obras Públicas -distribuidos en las distintas Subsecretarías y Direcciones- se conforman de una variedad de profesiones, la mayoría de ellas ligadas a la construcción: arquitectos, ingenieros, agrimensores, etc. Por su parte, la Dirección de Hábitat Social nuclea al equipo social de la Secretaría y posee, como función principal, acompañar todo el proceso de selección de los adjudicatarios: la realización del censo, la construcción de los indicadores para evaluar las situaciones socio-económicas de las familias, la confección de los listados para elevar a las autoridades, la realización de las actas de tenencia precaria, la definición de la ubicación de las familias en el predio construido, el acompañamiento en el proceso de mudanza, la organización de los espacios de interlocución pre y post mudanza. Por las características de estas tareas, la Dirección de Hábitat Social se conforma principalmente de trabajadores sociales, algunos de ellos estudiantes. Aunque también han pasado por la Dirección profesionales provenientes de otras disciplinas, como la Sociología y las Ciencias de la Comunicación.

Ahora bien, en este artículo analizaré la experiencia de presentación, y posterior censo, de un grupo de familias que habitaban en un territorio conocido como Villa Corina. He mencionado que el Municipio comenzó a implementar los Programas Federales en el año 2004 con Villa Tranquila como el primer territorio de implementación- y que luego fue ampliando sus acciones hacia otros barrios de Avellaneda. Esta incorporación de territorios implicó que los

\footnotetext{
${ }^{4}$ Este municipio es uno de los más antiguos del AMBA y se ubica en el denominado primer cordón. Hacia el norte limita con la ciudad de Buenos Aires -separada por el Riachuelo- al sudeste limita con Quilmes, al sudoeste con Lanús y al este con el Río de La Plata. Avellaneda comprende una superficie total de $55 \mathrm{Km} 2$ que abarca a las siguientes localidades: Avellaneda Centro, Dock Sud, Crucecita, Piñeiro, Gerli, Villa Domínico, Wilde y Sarandí. La mayor cantidad de población se concentra en estas dos últimas localidades. Si bien Avellaneda no posee la mayor densidad de población del AMBA, concentra un número significativo de ella debido a su cercanía con la CABA.

${ }^{5}$ En la actualidad, los proyectos de construcción de viviendas a cargo del Municipio son múltiples y variados. De acuerdo a la información publicada en noticias periodísticas locales, aquella que detallaron los funcionarios entrevistados y la que aporta oficialmente la Subsecretaria de Nación, Avellaneda -a Diciembre de 2012- lleva construidas cerca de 1900 viviendas, en proceso de construcción 1200 y licitadas 1500. Lo cual implica un total de 4600 entre las finalizadas y en proceso.
} 
responsables de la ejecución municipal fueran abordando todo un esquema de censos, realizados en su mayoría a través de una consultora privada. Estos censos tenían como finalidad realizar estimaciones acerca de las condiciones de vida de las familias de las diferentes villas y asentamientos de la zona y utilizar esa información en los planes de obra. Dentro de este esquema, el Municipio censó a todo el barrio de Villa Corina en el año $2005^{6}$.

Asimismo, el Municipio había definido cuatro líneas de acción para este territorio, combinando la información acerca de las características físicas de la zona con los recursos disponibles en el marco de los Programas Federales. A partir de allí, el gobierno local estableció como líneas de acción: la recuperación del conjunto urbano conocido como las torres de Villa Corina y la subdivisión del consorcio; la apertura de calles y la liberación de veredas; la regularización física y dominial de las manzanas y la construcción de viviendas para mudar a las familias de un sector conocido como los pabellones.

Dentro de esta última línea de acción, y en un lote sin poblar al interior del barrio, el Municipio planificó la construcción de un proyecto de viviendas y a partir de allí, algunas familias de Villa Corina comenzaron un proceso de disputa con el fin de conseguir ser incorporados como adjudicatarios en dicho proyecto.

En las líneas que siguen recorreré parte de esta experiencia de lucha para, a partir de allí, recopilar -desde el relato de los entrevistados- qué implican los procedimientos de recolección de datos -como el censo- en políticas de construcción de viviendas como la aquí mencionada.

\section{HISTORIA DE UN PROCESO DE ADJUDICACIÓN EN VILLA CORINA}

El barrio que aquí analizaré se ubica a dos cuadras del límite que divide a las localidades de Villa Domínico y Sarandí. Dentro de la primera localidad se sitúa el barrio conocido como Villa Corina, en el cual habitaban la mayoría de las familias que lo habitan actualmente.

Durante el año 2008, la empresa que emprendería la construcción del barrio ya había tomado posesión del lote y había comenzado tareas de desmalezamiento y preparación del terreno. Un grupo de mujeres, que representaban a 22 familias -todas ellas habitantes de los barrios de Villa Corina y el vecino barrio de Unidad y Lucha - resuelve ocupar el predio antes de que comenzara la construcción de las viviendas. Según cuenta una de las referentes, hacía tiempo que estaban buscando terrenos vacíos por la zona con el fin de ocuparlos y

\footnotetext{
${ }^{6}$ Cabe señalar que el plan de acciones para Villa Corina no ha quedado formalizado en un plan como sí en el caso del Programa de Urbanización de Villa Tranquila. Las entrevistas realizadas a funcionarios municipales que trabajaron hacia los comienzos de los Programas Federales en Avellaneda y a aquellos que trabajan aún en la actualidad, me ha permitido reconstruir las obras que detallaré a continuación.
}

Revista de Direito da Cidade, vol.07, nº1. ISSN 2317-7721 p.181-205 186 
autoconstruir allí sus viviendas. La mayoría de estas familias se encontraban habitando -en condiciones muy precarias- en de los pasillos de estos barrios, compartiendo la vivienda con otros grupos familiares, por lo cual, su solicitud de ser incorporadas a una lista de adjudicatarios se sustentará en esta condición de hacinamiento.

En noviembre del 2008 este grupo de familias ocupó el predio. Lo hacen, de acuerdo a lo expresado por una de ellas, contando con el apoyo de un grupo de camioneros que, hasta ese entonces, tenía el acuerdo de la empresa constructora para utilizar el predio a modo de estacionamiento. Pero al poco tiempo de haber ingresado, y encontrándose en tareas de desmalezamiento, los pastizales del lote comenzaron a incendiarse y debieren desalojarlo. La indignación que surgió en estas familias, luego de vivir este peligroso episodio, las llevó a dirigirse inmediatamente a la vivienda particular del intendente. Era un sábado a la noche y acamparon en la puerta hasta que éste las recibió el domingo por la mañana:

Nos quedamos ahí. A las 10 de la mañana él salió, así nomás, vestido con el short y una remera y... no le quedaba otra. Le planteamos el problema que teníamos, que éramos 22 familias que habíamos agarrado acá [se refiere al terreno donde hoy se asienta el barrio] pero era por necesidad porque ellos también dijeron que iban a hacer no sé qué y bueno, era todo un conflicto. Hasta que él agarró y nos dijo: Bueno, el lunes a tal hora se presentan en el municipio que yo los hago atender. (Entrevista a una habitante del barrio. Agosto 2011.)

Durante la audiencia del lunes, este grupo de mujeres le entregó al intendente un listado con 22 familias y obtuvieron el acuerdo de que serían adjudicadas en el barrio a construirse 15 de ellas. A partir de entonces, la Dirección debió llevar a cabo un censo a los fines de determinar quiénes serían las familias seleccionadas.

Por otra parte, un segundo grupo de familias llevó a cabo su propia presentación ante el municipio a los fines de lograr ser adjudicados en el mismo lote. A diferencia de las anteriores, estas se encontraban viviendo en una tira de casillas sobre una de las paredes del cementerio municipal. Una mujer de unos 50 años y un joven de 25 años, llevaron a cabo el armado de lo que denominaron su expediente. Lo interesante de esta experiencia es que -más allá de las dificultades que afrontaron para convencer y contar con el apoyo de sus vecinos- este expediente se organizó en torno a su problemática ambiental. Para estas familias, su estrategia de presentación consistió en detallar lo más exhaustivamente posible sus nefastas condiciones de vida, junto al paredón del cementerio municipal:

Nosotros buscábamos algo, queríamos buscar algo que apure, qué ponemos que apure... qué ponemos que apure... [Como pensando en voz alta] Y bueno vamos a meter a esos que tienen problemas... porque había muchos casos de enfermedad pero entraron esos dos nomás. Había muchos 
casos de enfermedad. Agarramos... es más sacamos fotos a las ratas... fotos a infecciones... nos subimos arriba del techo y les sacamos fotos a los cajones que estaban del otro lado del cementerio. Había un nene así todo brotado, le sacamos fotos... Todo lo que podíamos poner ahí... (Entrevista a habitante del barrio. Julio 2011)

Finalmente, el expediente incluyó a dos familias que no vivían sobre la pared del cementerio municipal, sino en un pasillo cercano. Estas dos familias se caracterizaban por presentar a algún niño con un problema de salud que se veía afectado por la exposición a las malas condiciones ambientales y requerían su urgente mudanza.

Luego de armar y presentar el expediente, pero principalmente de las insistentes solicitudes de entrevista al Intendente, estas familias lograron una audiencia y obtuvieron el compromiso de adjudicación de 25 de las viviendas construidas, previa realización de un censo.

Si bien la estrategia de presentación de este grupo de familias se sustentaba en problemáticas de salud y medio ambiente, su ubicación física aportaba otra ventaja al Municipio: su mudanza implicaría despejar una vereda del cementerio municipal, es decir, lo que en la jerga municipal se conoce como liberar un espacio público.

En ambos casos, las estrategias de demanda (Bourdieu, 1993) concluyeron con la promesa de adjudicarles una vivienda, a partir de la resolución del Intendente. El primer paso para que esta promesa se concretara era la realización de un censo.

En el siguiente apartado analizaré entonces la utilización del censo como el principal instrumento en la producción de adjudicatarios, priorizando el relato de los sujetos implicados.

\section{LA APLICACIÓN DEL CENSO}

Las principales vías de acceso a una vivienda de los Programas Federales en este Municipio son dos: la primera es cumplir la condición de habitar en una de las villas o asentamientos que se encuentran dentro de los planes de urbanización; la segunda vía es ser seleccionado dentro de un listado de familias en espera. A estas situaciones se las llama "de demanda espontánea". Para formar parte de este listado, las familias debieron entregar una nota vía la mesa de entradas del Municipio o bien, haber entregado una carta al intendente municipal, al gobernador de la provincia de Buenos Aires o la presidenta de la Nación, en alguno

\footnotetext{
${ }^{7}$ Utilizaré en este análisis la noción de estrategias elaborada por Pierre Bourdieu, dado que me refiero a aquellas prácticas que los sujetos llevan a cabo para conseguir ciertos fines dentro de un campo de disputas específico y en las cuales ponen en juego los capitales acumulados en luchas anteriores: "para que funcione un campo, es necesario que haya algo en juego y gente dispuesta a jugar, que esté dotada de los hábitus que implican el conocimiento y reconocimiento de las leyes inmanentes al juego, de lo que no está en juego, etc." (Bourdieu, 1993: 136).
} 
de los actos políticos oficiales.

Estas vías son el primer contacto con los profesionales de la Dirección de Hábitat Social del Municipio, quienes aplican variadas herramientas de intervención social para certificar y medir los argumentos que los sujetos presentaron, a fin de justificar su necesidad de acceder a una vivienda. Dichos instrumentos implican procedimientos de censo y posterior verificación ${ }^{8}$ en algunos casos- y una entrevista con posible informe social en otros, aunque a veces se combinan ambas herramientas ${ }^{9}$.

Las preguntas que intentaré responder en las líneas que siguen son:

¿Por qué el Municipio requiere del censo? Y ¿Por qué es el primer paso en el proceso de adjudicar una vivienda? Uno de los entrevistados me brindó la pista para esbozar una respuesta:

El censo es la llave (...) viste que en el Parque Indoamericano hubo gente que se tiró del tren para estar ahí... hasta una embarazada se tiró y la tuvieron que sacar en ambulancia. 0 sea la desesperación de la gente... la gente estaba ahí. iLos instrumentos que usás cómo impactan en la subjetividad de la gente! cómo la gente los apropia y cómo sabe que son llaves para entrar en la inclusión por decirlo de alguna manera... El censo es el legalizador por excelencia. O sea, no tenés censo, no existís... un barrio, un asentamiento, una fábrica tomada, un... lo que sea... el censo es lo que te hace aparecer y lo que les hace a las familias tener una esperanza de una futura vivienda. (Entrevista a un profesional de la Dirección de Hábitat Social. Mayo 2011)

Al momento en que se realizó el censo a las familias de Villa Corina, su realización se llevaba a cabo a través de dos modalidades: o bien se derivaba a una consultora privada, o bien se realizaba con personal de la Dirección de Hábitat Social. Las posibilidades de hacerlo de una u otra forma dependían principalmente de la cantidad de familias a censar.

Este procedimiento arroja la siguiente información: por un lado, un listado total de hogares que habitan un territorio -delimitado de manera previa a la aplicación del censo; por el otro, un detalle de los miembros de la familia, las condiciones habitacionales (cantidad de habitaciones y habitantes por habitación, servicios públicos, materiales de paredes, techos y pisos, etc.) y las características de su situación socio-económica desagregada en las dimensiones de trabajo, educación y salud. Una distinción importante al respecto de este procedimiento es

\footnotetext{
${ }^{8}$ La diferencia entre censo y verificación es que ésta última se aplica luego de transcurrido el censo con el fin de cotejar ciertos datos. En el caso de la construcción de viviendas, a verificar la composición familiar.

${ }^{9}$ Este conjunto de procedimientos o herramientas que los profesionales aplican a partir del encuentro con los sujetos demandantes, se organiza bajo la lógica de lo que Anthony Giddens conceptualiza como "sistemas expertos" entendiendo a estos como: "los sistemas de logros técnicos o de experiencia profesional que organizan grandes áreas del entorno material y social en que vivimos". En este caso el sistema experto está apoyado principalmente en el saber profesional de quienes componen la Dirección de Hábitat Social. El saber valorado es el que aportan las Ciencias Sociales para la medición y planificación de acciones ante diferentes problemas sociales. (Giddens, 1994: 37).
} 
que el censo desagrega -siguiendo la lógica del censo de población nacional- las viviendas de los hogares ${ }^{10}$, pudiendo éstos últimos ser muchos más, es decir, que una vivienda no equivale a sólo un hogar, con lo cual el número total de hogares es el dato más relevante a los fines de la planificación.

Estas descripciones permiten que el personal de la Secretaría de Obras Públicas definiera el número de viviendas requeridas para urbanizar un sector dentro de una villa o asentamiento, así como la cantidad de dormitorios por hogar. Los principales conflictos -al momento de realización del trabajo de campo- se generaban a raíz del tiempo requerido para la construcción de las viviendas, período durante el cual la composición de las familias -en muchos casos- variaba principalmente por el incremento de sus integrantes, lo cual conllevaba la creación de un nuevo hogar (que hubiera requerido contabilizar una vivienda más).

Pero además, es clave considerar que los habitantes de estos asentamientos conocen detalladamente estos procedimientos, porque el Estado, a través de diversos organismos, ha realizado censos una y otra vez. Esta reiteración de los censos, aunque se lleven a cabo con distintos objetivos, van dejando en ellos un repertorio ${ }^{11}$ acerca del cómo actuar ante estas situaciones así como los criterios que el Estado considera importantes en las decisiones posteriores a la realización del censo:

A mi hermano ya varias veces lo habían censado, con este fue la cuarta vez que le hacían el censo. Porque en ese momento él vivía con la señora y le dijeron: bueno, ustedes algún día ya van a tener la casita. Cuando se hicieron los

\footnotetext{
${ }^{10}$ En el censo opera un criterio de distinción entre vivienda y hogar. La vivienda refiere al espacio físico habitado, el hogar a los distintos grupos domésticos que habitan en ella. La distinción se realiza según quiénes comparten los gastos de alimentación.

${ }^{11}$ Esta noción de "repertorio" refiere a conocimientos prácticos que han sido incorporados en los sujetos a partir de sus experiencias de lucha previas y, a su vez, de la experiencia de otros sujetos en luchas que consideran similares. Para Charles Tilly esta categoría se vincula a procesos colectivos en los cuales: "la acción colectiva cae dentro de repertorios bien definidos y limitados que son particulares a diversos actores, a objetos de acción, tiempos, lugares y circunstancias estratégicas." (Tilly, 2000: 14). Estos conocimientos prácticos también se relacionan con lo que Bourdieu plantea en su conocido concepto de hábitus, que define como: "sistemas de disposiciones duraderas y transferibles, estructuras estructuradas predispuestas a funcionar como estructuras estructurantes, es decir, como principios generadores y organizadores de prácticas y de representaciones que pueden ser objetivamente adaptadas a su meta sin suponer el propósito consciente de ciertos fines ni el dominio expreso de las operaciones necesarias para alcanzarlos, objetivamente "reguladas" y "regulares" sin ser para nada el producto de la obediencia a determinadas reglas, y, por todo ello, colectivamente orquestadas sin ser el producto de la acción organizadora de un director de orquesta." Así mismo, el hábitus se vincula estrechamente la historia: "el hábitus origina prácticas, individuales y colectivas, y por ende historia, de acuerdo con los esquemas engendrados por la historia; es el hábitus el que asegura la presencia activa de las experiencias pasadas que, registradas en cada organismo bajo la forma de esquemas de percepción, de pensamientos y de acción, tienden, con más seguridad que todas las regias formales y todas las normas explícitas, a garantizar la conformidad de las prácticas y su constancia a través del tiempo." (Bourdieu, 1994:86-89).
} 
departamentos en Corina él ya estaba. Pero a mí me dijeron: mirá que tu hermano no está censado entonces ustedes también van a entrar, por intermedio de él. Ojalá, le decía yo, porque cuanto estuvo mi hermano nunca salió. Después yo me entero que en realidad sí salió en los departamentos de ahí, pero que lo rechazaron porque no tenía nenes, era solo. (Entrevista a habitante del barrio. Septiembre 2011)

Desde la lógica de los profesionales y funcionarios, el censo establece un número de viviendas a construir que no puede modificarse con el tiempo. Los conflictos surgen por los desfasajes que se producen con la obra pública, es decir, los tiempos de dinámica de crecimiento de las familias no se condicen con los tiempos de obra necesarios para construir las viviendas. Sin embargo, el censo opera congelando a las familias, quienes tienen que abogar por mantener su número de integrantes estable.

Este compromiso que se espera de las familias, opera como una obligación a cumplir dentro de un contrato que los sujetos asumen con el Municipio con el fin que se les adjudique una vivienda. En esta clave, Vanina Lekerman (2010), en un reciente trabajo acerca de las redes de relaciones que se construyen entre los habitantes de las villas de la Ciudad de Buenos Aires y los funcionarios, plantea que el Estado aplica en estos espacios de negociación la lógica del don y el contra don. Esto implica que la vivienda opera como un objeto de negociación: el Estado local la otorga a ciertos habitantes y establece una serie de obligaciones que se espera que ellos cumplan.

Pero además, el censo y la verificación son las herramientas primordiales en el proceso de producir consenso. Una de las profesionales entrevistadas describía de la siguiente manera este último proceso:

Entonces cuando a nosotros nos piden eso, ponerle que tenemos el plano acá, nos dicen: este sector necesitaríamos liberarlo para empezar a construir casas acá entonces ¿vos qué necesitás hacer? Tenés que ir al terreno a confirmar quién vive, la cantidad de personas que viven por vivienda, si hay ampliaciones de familia, cuantos grupos... entonces nosotros hacemos las verificaciones de ocupación ¿̀ qué hago con eso? Congelo otra vez la población y después mandás a pedir el prototipo... (Entrevista a un profesional de la Dirección de Hábitat Social. Febrero 2012)

Desde los relatos de los profesionales entrevistados, es posible establecer que herramientas como el censo y las verificaciones, generan dos efectos productores de realidad en los sujetos destinatarios: por un lado, un efecto legitimador, dado que operan otorgando existencia social; por otro lado, un efecto cristalizador, en cuanto establecen una composición oficial de la familia, que se debe mantener intacta hasta tanto se concluyan las viviendas.

A diferencia de la visión planificadora de los funcionarios, gran parte de los profesionales entrevistados se refirieron a las decisiones de la Secretaría -en cuanto a la 
planificación- como producto de una lógica errática, en la cual un territorio cobra interés por motivos que varían según las situaciones ${ }^{12}$ pero cuya explicación requeriría de un análisis que no es tan evidente para ciertos actores, como por ejemplo, para los profesionales que salen a censar. Por lo cual, muchas veces los motivos de por qué censar un territorio no son explicitados y la explicación que esbozan los profesionales es que, por alguna razón, el Municipio -encarnado en sus funcionarios- señala con su dedo en esa parte del territorio:

Volvemos a lo mismo, esto de la falta de una estrategia planificada. Pasó con [menciona a uno de los territorios del municipio]... o sea depende de la emergencia que surja o qué sé yo... matan a alguien en un asentamiento y es un asentamiento peligroso bueno, hay que censar ese asentamiento... hay que abrir una calle en tal lado porque... andá a saber... ni siquiera nosotros sabemos cuál es el criterio último de por qué [El Intendente] puso el dedo en esa parte del mapa. (Entrevista a un profesional de la Dirección de Hábitat Social. Mayo 2011)

En una coyuntura específica, ambos grupos de familias, y a partir de diversas estrategias, lograron constituirse como solicitantes legítimos a partir de una "estrategia de visibilidad". Estas apuntan -como su nombre lo indica- a volverse visibles para el Estado, es decir, lograr que éste los contemple como posibles adjudicatarios de una vivienda estatal en detrimento de otros. Así mismo, estas estrategias, presentan una lógica inversa a la planteada por María Carman (2006) para el caso de los ocupantes en el barrio del Abasto. En este caso, los habitantes desplegaban distintas estrategias para volverse invisibles ante el Estado que consistían en, por ejemplo, tapiar sus viviendas. Esto les permitía hacer invisible el hecho de que ocupaban un edificio del cual no eran propietarios, dado que esto pondría en evidencia una práctica que el Estado condenaba por considerar ilegal.

En resumen, y en palabras de un entrevistado, los motivos que llevaron a que estas familias -y no otras- habitaran este barrio, respondería a la siguiente secuencia de hechos:

Ahí hubo una serie de conjugación de cosas me parece [refiriéndose al barrio] Por un lado el Intendente quería abrir esa calle del cementerio, que eran los 50 metros posteriores, pero cuando se fue a ver [se refiere a los profesionales de la Secretaría] se vio que eso estaba peor y las familias armaron el expediente justo en ese momento $y$ entonces dijo el Intendente hay que hacer algo con esta calle... y apareció el expediente y dijo hay que hacer algo con este expediente. (Entrevista a un profesional de la Dirección de Hábitat Social. Mayo 2011)

Una vez que el Municipio resuelve censar un territorio se producen invariablemente efectos. Los habitantes saben que el censo es la llave -como bien señalaba uno de los

\footnotetext{
12 Me refiero a situaciones como: la protesta de un grupo de vecinos en la vía pública, la difusión de ciertas poblaciones en condiciones precarias de vida en los medios de comunicación, etc.
} 
profesionales entrevistados- y que ese marco de oportunidad puede no volver a repetirse por mucho tiempo o quizás nunca más.

Así como en un primer momento de sus reclamos la estrategia se apoyaba en volverse visibles para el Estado, una vez definida la adjudicación, la estrategia tendrá que ver con obtener el prototipo de vivienda que resulte más favorable en relación con la composición familiar. Esto implica, por ejemplo, que en una familia con hijos mayores que viven con su pareja y quizás sus hijos, éstos sean contemplados como un hogar aparte, es decir, que requerirán una vivienda independiente. En muchas ocasiones esto se extiende a hijos mayores que potencialmente formarán una familia en el futuro y necesitarán una vivienda propia. Pero más allá del detalle de las situaciones particulares, lo cierto es que ante este marco de oportunidad, los habitantes del asentamiento a censar, despliegan estrategias para sacar provecho de esta instancia ya que, como mencioné anteriormente, conocen detalladamente que luego no podrán introducir modificaciones en la composición familiar que quedó cristalizada.

Desde la perspectiva de los funcionarios y profesionales municipales, esta especulación al momento del censo los pone ante el dilema de cómo comprobar quienes viven realmente en cada vivienda: las mentiras y las verdades se vuelven su objeto de análisis y, principalmente para la Dirección de Hábitat Social, se constituye en su tarea específica apoyada en el expertise profesional de las Ciencias Sociales y más específicamente, del Trabajo Social:

Entonces más o menos a ojo, tratando de ver ahí que fuera lo más justo posible yendo a verificar la famosa cuestión de los engaños [se refiere al caso específico de un barrio en la localidad de Wilde] vos tenías que tener el buen sentido de ver si una familia vivía ahí o era una familia traída de no sé dónde. Y las observaciones... otra cosa medio de vigilante ¿viste? Pero era verdad, no queda otra, no sé que en realidad... [Pensando en alternativas a este procedimiento] la que queda es un buen laburo barrial (...) Pero la realidad es que el censo estaba infladísimo y las organizaciones colaboraron pero como son gente del barrio, también les cuesta a veces decir 'mirá que esta familia no es de acá'. (Entrevista a profesional de la Dirección de Hábitat Social. Noviembre 2012)

Entonces, los habitantes tienden a inflar el censo y en consecuencia el Municipio sobrecensa, pero los profesionales y funcionarios tienen conocimiento de estas estrategias que se despliegan en los territorios y tienden entonces a desinflarlo, es decir, a reducir el número total de hogares. Esta tensión se mantiene durante todo el procedimiento porque, en primera instancia -como señalaba uno de los entrevistados- los vecinos saben que todos los integrantes de la familia deben estar ahí el día del censo, porque luego no serán considerados: 
me dicen: '¡Delia! van a ir unas chicas que van a hacer un censo por unas viviendas iUh qué bueno!' [Se ríe]. (Entrevista a una de las habitantes del Barrio. Septiembre 2011)

Los habitantes conocen las reglas de juego y, en consecuencia, se preparan para el día del censo. Para los profesionales muchas veces el censo inflado o el sobrecenso es una consecuencia del censo avisado:

El censo en Corina (que era un terreno re áspero) lo hicimos súper rápido y le dimos los resultados a la semana al intendente. [Se refiere a un censo que se realizó de manera posterior a la entrega del barrio con motivo de abrir la calle que continuaba una de las veredas del cementerio municipal]. Cuando nosotros hicimos el primer censo se nos duplicó el número de familias que pretendíamos obviamente porque se regó la bola y se sobre-censó. Sobrepasó mucho los números que él esperaba [se refiere al Intendente]. Como fue un censo avisado, se notaba que había mucha gente que venía de las torres de Corina o de otros barrios que se habían instalado ese día a estar ahí y a sumarse para el plan de urbanización. (Entrevista a profesional de Hábitat Social. Junio 2011)

Como mencionaba uno de los profesionales entrevistados, un trabajo sostenido en el barrio -lo que al interior de la Dirección se conoce como hacer sede-permite llevar a cabo una tarea tendiente a cotejar y limpiar los resultados de un censo inflado:

Vos tenés que mudar un lugar y sabés que tenés que hacer el censo, haciendo sede vos podés hacer una limpieza de ese censo, hacer una cosa más organizada y más prolija. (Entrevista a profesional de Hábitat Social. Junio 2011)

Como ya mencioné, luego de la realización del censo el Municipio cierra la posibilidad de incorporar nuevos integrantes en la composición de las familias pero en algunos casos (como en la urbanización de Villa Tranquila) transcurre tanto tiempo entre la realización del censo y las mudanzas que los profesionales vuelven al territorio a verificar a las familias censadas. A diferencia del censo, la verificación tiene la característica de ser una especie de sondeo rápido de la situación, como mencionaba una profesional entrevistada, ante la urgencia de actualizar los datos para entregar una obra de viviendas, la prioridad en las tareas de la Dirección es salir a hacer censos y verificaciones relámpago.

Si bien algunos habitantes aprovechan la instancia del censo y le presentan al Municipio una conformación familiar inflada, otros se resisten a ser censados, como sucedió en el caso del barrio analizado. Pero también porque luego de años de habitar en el lugar y de haber sido censados varias veces por distintos gobiernos e incluso para diversos fines ${ }^{13}$, los habitantes no

\footnotetext{
13 Los programas de construcción de viviendas, como los aquí mencionados, no son los únicos que presentan dentro de su metodología de implementación la necesidad de la realización de un censo. Otro tipo de políticas, como por ejemplo las alimentarias, también utilizan estos mecanismos. En algunas
} 
creen que la mudanza pueda llegar a suceder. En otros casos, en cambio, ellos acceden a ser censados y toman conocimiento de las reglas en cuestión pero manifiestan su descontento al respecto, como mencionaba uno de los habitantes entrevistados:

Estábamos ahí, vinieron, nos censaron y bueno, en ese tiempo ellos no estaban viviendo conmigo [se refiere a parte de sus hijos] pero después, cuando nos mudamos, se vinieron a vivir conmigo. Nos vinimos a vivir acá porque si no habría sido una casa más grande [es decir, si sus hijos hubieran estado viviendo allí durante el censo les hubieran asignado una vivienda con más dormitorios]. Había problemas porque supuestamente no permitían, decían que no nos permitían meter más gente en la casa pero yo... en mi casa puedo meter a quien quiera. (Entrevista a habitante del barrio. Septiembre 2011)

El meter gente en la casa para inflar el censo, el estar ahí, el avisar o no avisar, elucidar las mentiras y las verdades son todos puntos conflictivos en este proceso. Los sujetos desigualmente posicionados- llevan a cabo la estrategia que les resulta más conveniente apoyándose en sus experiencias previas en su relación con el Estado y su corpus de conocimiento al respecto de estas interacciones ${ }^{14}$. Un reciente trabajo acerca de la urbanización de la Villa Rodrigo Bueno describe claramente este universo de sujetos y tensiones en torno al censo:

Algunos habitantes de la villa se resistían a ser censados continuamente. $Y$ otros cuestionaban el universo de población abarcado por el censo alegando intereses espurios $u$ omisiones. Las trabajadoras sociales tendían a leer la presencia de todo eventual nuevo integrante del grupo familiar como una estrategia para aumentar el monto del subsidio (...) No obstante la villa seguía creciendo, especialmente durante aquellos meses en los que el desalojo parecía inminente. (Carman, 2011: 67-68).

Pero el censo no sólo provoca efectos en la población censada, sino también en aquella que queda por fuera de este proceso y que presenta condiciones materiales de vida similares a la población que resultó adjudicataria:

Cuando la gente se entera que hay un barrio empiezan a

ocasiones, los vecinos realizaban a los profesionales de Hábitat la siguiente pregunta: ¿Para qué me estás censando?

${ }^{14}$ Este corpus de conocimiento acerca de cómo actuar, históricamente construido, se vincula con el ya definido concepto de hábitus. 
venir acá: che yo sé que van a construir en... y acá están anotando. (...) nunca te va a pasar que tenés un barrio y decís... che, ¿A quién se lo doy? [Es] al revés, porque siempre pasa que la gente se va enterando, van viniendo, van armando y te armás una lista que nunca te alcanza [para] satisfacer. (Entrevista a un funcionario municipal. Septiembre 2012)

Las veinte familias que formaban parte del expediente fueron censados por los profesionales de la Dirección, pero aquellas familias cercanas, asentadas de manera contigua a las primeras -que viven en las mismas o incluso peores condiciones de habitabilidad- no fueron contempladas en el proceso. Esta situación provocaba la reacción de estos otros habitantes que expresaban su descontento por no haber sido considerados, y exigían serlo:

Yo hago referencia concretamente a esa franja siguiente, que era la franja que continuaba sobre el cementerio... Lo primero es que claramente (la gente que se queda) tiene esto de 'Por una casita no me tocó a mí, por un metro no me tocó a mí' y esa cosa de '¿Por qué a ellos sí y a mí no?'. (Entrevista a profesional de Hábitat Social. Junio 2011)

En los casos más extremos -aunque cabe señalar que han sido los menos- en los barrios en los cuales el Municipio censó a algunos pobladores para un proyecto de viviendas -dejando fuera a otros- unos días antes de su entrega definitiva, las viviendas casi finalizadas fueron ocupadas por los vecinos aledaños ${ }^{15}$. En estas situaciones, el Municipio llevó a cabo un procedimiento de desalojo que permitió expulsar a los ocupantes que no habían sido validados a través del censo y, una vez expulsados, concretó la mudanza de los sí censados. Entonces, las poblaciones no sólo se vuelven visibles para el Estado y cobran existencia a partir del censo, sino que esta validación invalida a todos aquellos que quedaron por fuera de este procedimiento. Establece, por lo tanto, un recorte entre quienes son considerados los legítimos adjudicatarios y quienes quedan en el plano de los ilegítimos; al extremo de utilizar una herramienta como el desalojo para validar y sostener esta decisión. Desalojar, expulsar, es la acción que se encuentra en el polo opuesto a resolver las condiciones habitacionales de las poblaciones ${ }^{16}$.

Antes de concluir con la experiencia de adjudicación del barrio en estudio, mencionaré algunos aspectos referidos a otro de los instrumentos frecuentemente utilizados en la producción de adjudicatarios: el informe social.

\section{EL INFORME SOCIAL EN LA PRODUCCIÓN DE LOS ADJUDICATARIOS}

15 La ocupación de viviendas próximas a ser entregadas, por parte de quienes no resultaron adjudicatarios, es una estrategia recurrente en distintos territorios del AMBA, principalmente en los proceso de urbanización de villas y asentamientos en la Ciudad de Buenos Aires.

16 En este sentido, Carman se refiere a las "esquizopolíticas" del Estado, al identificar que las prácticas oficiales pueden combinar tanto acciones asistencialistas como represivas: "el gobierno local consagra, en fin, las políticas de lo efímero y puede tanto proteger y dar asilo, como despojar y condenar." (Carman, 2011: 194). 
En la Dirección de Hábitat Social, el informe social era un instrumento utilizado para la comprobación de las situaciones detalladas en algunas cartas y notas que pasaban a integrar el listado de demanda espontánea. No era de tan frecuente realización como las verificaciones y censos pero, de acuerdo al relato de los funcionarios entrevistados, a partir del año 2010 su uso se incrementó con el fin de cotejar las situaciones de las familias que le entregaban las cartas al intendente.

Tanto el censo como las verificaciones, pero principalmente los informes sociales, tenían como fin identificar y recortar una necesidad habitacional comprobable, es decir, constatar el relato descripto por los sujetos solicitantes y establecer la veracidad de su solicitud de vivienda:

Vamos a hacer un informe social, a constatar digamos las condiciones habitacionales para ver cómo viven. (Entrevista a profesional de la Dirección de Hábitat Social. Febrero 2012.)

Los requisitos dependían también del proyecto de vivienda. Creo que la población a la que estaba orientada estaba marcada por la ubicación del proyecto de vivienda pero bueno, concretamente [la condición] era que hubiera una necesidad habitacional comprobable, real donde fueran familias que realmente no tuvieran la posibilidad de solucionar su problema habitacional por otros medios. Había casos de familias con personas discapacitadas o enfermedades crónicas y donde las condiciones habitacionales afectaban esa situación. (Entrevista a profesional de la Dirección de Hábitat Social. Agosto. 2012)

Para la aplicación de estos instrumentos, los profesionales se trasladaban hasta el domicilio a fin de cotejar sus condiciones de vida y evaluar si ese relato encajaba dentro de lo que el Municipio definía como necesidad habitacional. Ahora bien, a diferencia del censo y la verificación, que también implica un acercamiento de los profesionales al territorio, el informe social enfatiza aún más la condición individual del pedido, dado que no se aplica a todas las situaciones presentadas ni frente a un pedido colectivo. Este instrumento priorizaba el análisis minucioso caso por caso y considerando la urgencia:

Hoy en día los informes que voy a hacer son los que consideramos que presentan más urgencia a nivel salud o habitacional, [esos casos] que vos decís: escuché algo que amerita que vayamos a hacer un informe (...) El informe social es un instrumento más de laburo y nosotros decidimos cuando lo hacemos, no es algo tipo trabajo en serie, distinto es una verificación. (Entrevista a profesional de la Dirección de Hábitat Social. Febrero 2012.)

La comprobación de las necesidades habitacionales es el mayor potencial del informe social. El profesional deja asentando de qué modo vive esa familia y si amerita ser beneficiario de una de las viviendas que construye el Municipio. El informe social se constituye entonces 
como otro instrumento más que contribuye a dilucidar las verdades y las mentiras, tomando como punto de partida los relatos y la documentación que presentaban los sujetos.

En resumen, partiendo de estas solicitudes de viviendas, los profesionales aplican una combinación de instrumentos de medición y verificación de las necesidades habitacionales tantas veces como sea necesario hasta lograr arribar a una conformidad al respecto de la información obtenida. Es decir, que no solo se aplican variados instrumentos sino que también dicha aplicación puede reiterarse tantas veces como sea necesario:

Lo que yo creo es que cuando tenés que seleccionar familias, lo que tenés que decir es "Bueno, es a ver tengo una casa y tengo 10 familias". Nosotros, lo que hemos profundizado, que se ha sumado muchísimo trabajo, es que hacemos entrevistas y entrevistas y entrevistas; informe socio ambiental; pedimos documentación y no me quedó claro y te vuelvo a llamar y volvemos a ir a tu casa. Y entonces, en un momento tenés las 10 historias desarrolladas y medís técnicamente. Porque también, lo que hemos podido como blanquear, es: Nosotros estamos seleccionando una familia, tenemos que hacer una selección técnica, acá no hay pobrecitos los pobrecitos, porque si no, te entrampás en una situación de beneficencia. (Entrevista a funcionaria municipal. Noviembre 2012)

Desde la perspectiva de los profesionales, el informe social se presenta como un instrumento eficaz en la descripción de las condiciones de vida de una familia y esto les provee una información que les permita tomar una decisión objetiva y desplazar, al momento de realizar su recomendación, aquellos elementos que consideran como más cercano a lo afectivo ${ }^{17}$.

Pero este instrumento tiene además otra utilidad, dado que contribuye a desarrollar una estrategia de visibilidad, que en este caso parte desde el Estado. El informe social implica que los trabajadores sociales caminen el barrio y esto permite que los habitantes contemplen la acción estatal a través de sus profesionales:

Y que después, en persona, venga un trabajador social. [Se refiere al circuito de las cartas entregadas en mano al intendente]Es de muy fácil visualización porque vos estás en un barrio una mañana, dos o tres personas caminando, una cierta cantidad de horas haciendo el recorrido de visitar a las viviendas que estaban más cercanas, con una ficha en la mano y te ven a la legua que sois trabajador social, entonces se empieza a correr esa bola en el barrio (...) Era bien un

\footnotetext{
${ }^{17}$ Con esta expresión nativa, retomo algunas cuestiones mencionadas por los profesionales durante las entrevistas. De acuerdo a sus relatos, existirían una serie de criterios sobre los cuales se basa la decisión de adjudicar que no poseen una apoyatura científica sino más bien afectiva. Un ejemplo serían aquellas situaciones en las cuales los profesionales definen a quién adjudicar una vivienda por el hecho de poseer un registro cotidiano del caso, dado que el postulante se acerca recurrentemente a la Dirección. A esta decisión la han mencionado como aquella que se sustenta en el hecho de tener más presente una cara que otras. Podemos inferir que en estas interacciones cotidianas también estarían implicados, como elementos afectivos, cuestiones más ligadas a la empatía de los profesionales hacia ciertos solicitantes.
} 
trabajo del visitador social (...) Y se empezaba a divulgar en el barrio y éramos perseguidos por las personas! (...) Pero su objetivo lo logran, si ellos querían visualizarse, se visualizaron seguro. (Entrevista a una profesional de la Dirección de Hábitat Social. Junio 2012)

El informe social es la herramienta que más polémica generó entre los profesionales entrevistados, principalmente entre quienes son trabajadores sociales. Si bien por un lado algunos señalaron su potencial como instrumento de control (dado que aporta información clave para discernir las verdades de las mentiras), la mayoría enfatizó su carácter individualista, dado que refuerza la metodología de trabajo por caso, en detrimento de una metodología más grupal o comunitaria ${ }^{18}$ :

Por ejemplo, en Corina, era ir a ver esta casa sí y esta casa no, no importaba que esa casa estuviera también hecha pelota. Lo que nosotros veíamos es que todas eran para priorizar. Si ellos querían hacer un trabajo con Corina la forma era otra: un trabajo sostenido, articulado con referentes y hacés un barrido general, como se venía haciendo antes. En vez de ser un trabajo continuado y un proceso en el que se avanza, era una cosa esporádica. (Entrevista a una profesional de la Dirección de Hábitat Social. Junio 2011)

Pero además, esta apuesta de la gestión de los años más recientes por realizar un trabajo caso por caso en detrimento de un trabajo barrial, tendría otra intencionalidad que implica todo un perfil de vinculación entre los funcionarios y los sujetos que les solicitan una solución a sus problemas. Para uno de los profesionales entrevistados, este modo de vinculación refuerza el personalismo y, podríamos agregar, cierto paternalismo:

Eso es lo que se termina fomentando: se fortalece a la vez la potencia de la carta. Un circuito mágico en el que vos tenés que hacer algo de persona a persona. Las cartas que llegan son las cartas que se le entregan al intendente en persona, a la presidenta en persona y que después, en persona, venga un trabajador social, que es la persona profesional. [Es] esta cosa de volver a fortalecer el personalismo. Y si ellos querían fortalecer el personalismo dándole vivienda a los que le daban la carta al intendente, ya está: "Yo le entregué mi carta al intendente, así que si vos querés tener tu casita entregale la carta al intendente y así vas a conseguir la casa". No porque una comunidad se movilizó, se organizó o porque un grupo de vecinos entregó una carta y hace ver su

\footnotetext{
${ }^{18}$ En el campo del Trabajo Social, la formación curricular -al menos en la Universidad de Buenos Airesrealiza una distinción entre tres niveles de intervención, que operan a modo de recortes de la realidad social. El primero es a nivel comunidad, el segundo grupal y el tercero familiar. En este último se contempla la realización de un informe social. Si bien la currícula de formación de las distintas unidades académicas ha sufrido modificaciones -de hecho la Universidad de Buenos Aires lo ha hecho recientemente- esta distinción entre caso, grupo y comunidad opera muy fuerte en el imaginario de los Trabajadores Sociales y parte de la crítica que realizaron algunos de los profesionales entrevistados al modo de intervenir desde la Dirección, se sustenta en esta división histórica del campo disciplinar.
} 
situación de necesidad. (Entrevista a una profesional de la Dirección de Hábitat Social. Junio 2011)

El personalismo no es algo nuevo dentro del universo de las políticas públicas. Algunas instituciones que han dejado su impronta en la Argentina-como la Sociedad de Beneficencia o la Fundación Eva Perón- "institucionalizaron" algunos aspectos que aún hoy podemos encontrar en los modos en que se implementan las políticas públicas. Pero lo más relevante de esta tendencia es que las acciones estatales que se encuadran, en este caso, dentro de la política de construcción y entrega de viviendas municipal, tienen como objetivo establecer el registro en los sujetos destinatarios de quién es el funcionario que se encontraba a cargo al momento de la adjudicación, de manera tal de reforzar en su imaginario a quién deberán estarle agradecidos. Nuevamente, en la lógica del don y contra don que mencionaba anteriormente, la vivienda es un objeto de intercambio y negociación.

Finalmente, y antes de culminar este artículo, destinaré las líneas que siguen a relatar cómo se dirimió, para cada grupo de familias demandantes, la definición de sus listados de adjudicatarios.

\section{EL CIERRE DE UN ACUERDO}

Con la información aportada por el censo realizado a ambos grupos de familias, los profesionales y funcionarios de la Dirección pusieron en común quiénes serían los adjudicatarios. En ambos casos implicó hacer un recorte, dado que se había preestablecido un número máximo de viviendas para cada listado.

Las herramientas aplicadas fueron diferentes en cada caso. Para el segundo grupo aquel que armó un expediente con problemáticas de salud y medio ambiente - la estrategia consistió en la realización de una asamblea en el barrio, con todas las familias presentes, en la cual el personal del Municipio puso en común los resultados del censo y pidió, a través de la confección de un acta que firmaron todos los vecinos, el acuerdo unánime -y escrito- acerca del listado final de familias. Como este acuerdo no se logró en la primera instancia debido a las acusaciones cruzadas acerca de quiénes vivían realmente o no allí, el Municipio repitió este esquema y se realizó una nueva asamblea -con unos días de diferencia- en la que finalmente se llegó a un acuerdo. Bajo esta modalidad, la definición de quiénes eran las familias se depositaba en el propio grupo. El criterio que fundamentaba esta decisión era que ellos mismos sabían quienes vivían y quienes no vivían realmente alli ${ }^{19}$.

\footnotetext{
19 En el lapso entre la realización de la primera asamblea y la segunda, el Municipio depositó en los sujetos la responsabilidad de acordar un listado definitivo de familias. En ninguna entrevista de las realizadas se mencionó en detalle qué argumentos se discutieron y qué hechos transcurrieron en estos
} 
Para el primer grupo, la estrategia municipal fue diferente dado que estas familias se localizaban en diferentes puntos de Villa Corina y del vecino barrio Unidad y Lucha. Es así que para la definición de este listado se resolvió aplicar criterios en consonancia con los utilizados para los casos de demanda espontánea.

Finalmente, el Municipio estableció que destinaría al primer grupo de familias veinticinco de las viviendas construidas y a las otras sólo cinco en este proyecto. Las restantes diez deberían esperar a la construcción de las viviendas que estaban previstas en otros proyectos cerca de Villa Corina. Esta resolución da cuenta de que los criterios puestos en juego por el primer grupo de familias -sobre todo la posibilidad de liberar una vereda a partir de su mudanza- cobraron más importancia que el aliviar la situación de hacinamiento del otro grupo.

Una vez consensuados los listados de adjudicatarios, cada familia recibió un acta de beneficiario (en un acto organizado para tal fin) y comenzó un período de espera hasta que las empresas concluyeran la construcción de las viviendas.

\section{CONCLUSIONES}

La aplicación de técnicas de recolección de datos, como los censos, las verificaciones y el informe social, son frecuentemente utilizados en las políticas habitacionales como la aquí mencionada. El análisis de un caso de su utilización, desde una perspectiva etnográfica, nos permite adentrarnos en el complejo tejido de sujetos y relaciones que conlleva su aplicación: los criterios que finalmente sustentan la decisión de adjudicar una vivienda no son idénticos para cada territorio, y los sujetos que habitan estos barrios toman conocimiento de las reglas de juego -estar ahí, inflar el número de integrantes- y las manipulan a su favor.

En las líneas que siguen, me enfocaré en señalar los que considero como los aspectos principales para promover una reflexión crítica acerca de estos instrumentos de medición y producción de los adjudicatarios en las políticas habitacionales.

En primer lugar, cabe señalar que, además de propiciar una primera distinción entre legítimos e ilegítimos adjudicatarios, en la aplicación de estos instrumentos opera en cierto modo la lógica del merecimiento. En el reconocido trabajo Merecer la ciudad, Oscar Oszlak realiza un análisis de las políticas urbanas aplicadas durante la última dictadura militar. La lógica del merecimiento operaba en el sentido de establecer un esquema elitista acerca del habitar la Ciudad de Buenos Aires. En este sentido, el Estado definía un modelo de poblador deseado y expulsaba a aquellos que no lo correspondían. Años más tarde, durante la década de los '90, se produjo el auge de las reconocidas "políticas focalizadas". Estas políticas conllevan 
necesariamente un recorte de la población destinataria de sus acciones, y en este proceso construyen un modelo de los sujetos destinatarios que, a la vez que incluye a algunos, expulsa a otros.

Considerando que las condiciones materiales de vida de las poblaciones de territorios como los aquí analizados son similares y que el derecho a la vivienda -incorporado a la Constitución Nacional de la Argentina- proclama la universalidad del mismo, la política estatal opera validando distinciones entre los pobladores y, al hacerlo, define no sólo sujetos merecedores de sus acciones, sino también un estándar acerca de cuáles son las pautas para acceder a este merecimiento.

El censo excede la simple recolección de datos y es mucho más que una herramienta estadística. No sólo está en juego en este proceso la acumulación de información sobre las condiciones de vida de cierto grupo de familias, de manera tal de justificar las decisiones políticas, sino que también, y principalmente, es un proceso que se sustenta y nutre del universo de interrelaciones que se suscitan entre los actores implicados. En línea con lo que plantean Manzano y Moreno:

Censar implica activar vínculos cotidianos entre referentes sociales y políticos con los pobladores de sus zonas de influencia, a través de convocatorias a reuniones o diálogos permanentes para convencer a aquellos [y] que resisten la incorporación a registros censales. Censar también representa una oportunidad para afirmar y visibilizar la tarea de referentes locales en el acercamiento a profesionales (médicos, trabajadores sociales, maestros) y funcionarios estatales. Censar es producir de manera conjunta y disputada con agentes estatales categorías para la intervención política -como la familia/vivienda o desnutrición/desocupación-que se acomodan parcialmente a los requerimientos de programas estatales, a la vez que los recrean y redefinen. (Manzano \& Moreno, 2011: 9).

En segundo lugar, cabe considerar que el lenguaje que los sistemas expertos utilizan para describir la realidad, como bien señalan Fonseca y Cardarello (2005), forma parte de un "sistema de clasificación" que, a la vez que incluye a ciertos sujetos bajo determinadas categorías, excluye a otros. Estos mecanismos de inclusión y exclusión se producen en simultáneo. Así, al formular que las viviendas a construir serán destinadas a ciertos sujetos, el Municipio establece que no serán para todos y realiza entonces un recorte entre potenciales adjudicatarios.

Para Talal Asad, las acciones de los Estados modernos se fundamentan en la formulación de derechos -legalmente promulgados- y en las nociones de libertad e igualdad, propias de la doctrina del liberalismo. La perspectiva de individuos libres e iguales favorece un posicionamiento hacia los sujetos a partir del cual todos son equivalentes $y$, por lo tanto, 
indiferentes para el Estado pero, como bien advierte el autor: "Aún cuando los individuos son tratados como verdaderamente equivalentes, un burócrata puede juzgarlos como se les plazca."(Talal, 2008: 56).

Será la decisión de los burócratas -en el caso de estudio los funcionarios- en las diversas instancias de decisión, quiénes juzguen a los sujetos y los clasifiquen de acuerdo a su perspectiva e intereses.

Entonces, y en tercer lugar, los argumentos que fundamentan el cómo juzgar a los sujetos son decisiones de los burócratas de turno -funcionarios de carne y hueso- que establecen no sólo qué criterios tienen validez para recortar a las poblaciones que solicitan el acceso a una de las viviendas estatales, sino también toda una modalidad de relacionamiento con ellas. Un aspecto que se presentó recurrentemente en el relato de la mayor parte de los profesionales -y también en el de algunos funcionarios municipales- es que en estos casi diez años de implementación de los Programas Federales en el Municipio pueden identificarse dos modalidades de relacionamiento. La primera -más grupal- implica el trabajo sostenido en un territorio haciendo sede, lo cual facilita un conocimiento más detallado de las condiciones de vida en un espacio de la ciudad, con la consecuente disponibilidad de mayor información para la toma de decisiones, así como la posibilidad de abrir instancias de trabajo colectivo, como mesas de trabajo por barrio. La segunda modalidad, se enfoca en el tratamiento individual de las solicitudes de viviendas que se traducen en incontables cartas o notas elaboradas individualmente. Más allá del énfasis que cada gestión de gobierno pueda poner en una u otra modalidad, siempre operan ambas en conjunto. Algunos autores consideran que el tratamiento de "casos particulares" es algo inherente a los modos que asume la implementación de las políticas habitacionales, al menos en el AMBA. En un interesante trabajo denominado "Vecinocracia" (2011), los autores analizan el caso de la toma del Parque Indoamericano y realizan la siguiente reflexión, que bien puede aplicarse al análisis del caso que aquí nos interesa:

En un contexto donde hay riquezas "para repartir", este tipo de acciones logran establecer rápidas negociaciones con las esferas oficiales, preocupadas por pacificar el conflicto, abriéndose rápidamente una negociación entre las partes. En este esquema, las personas refieren menos su situación a una escena de lucha y organización colectiva y más a una situación personal o familiar. Y desde las instancias oficiales se trata menos de lidiar con referentes políticos orgánicos y representativos y más de establecer casos particulares. De allí la recurrencia al censo como primera y principal operación organizadora de la negociación. (...) Una vez abierta la negociación, la capacidad de acción se transforma 
en demanda o 'caso' capaz de encuadrarse como "caso social". (Colectivo Simbiosis Cultural, 2011: 20)

La construcción del "caso social" es, desde esta perspectiva, la respuesta frecuente a los conflictos -como la toma de tierras- que se suscitan en los diferentes territorios. Entre el conflicto $^{20}$ por adquirir una vivienda y su resolución, media el conocimiento técnico.

Por lo tanto, y a modo de cierre, cabe afirmar que todos los instrumentos que se mencionaron en este apartado -el censo, la verificación y el informe social - forman parte de un conocimiento práctico y experto acerca de cómo abordar este tipo de problemáticas que el Estado ha forjado a través de años de políticas en el campo del hábitat y la vivienda. Su repertorio de acción se nutre del saber experto de variadas disciplinas, principalmente de las Ciencias Sociales, que tienen la capacidad de sumar sus modos de ver, decir y actuar ante variadas situaciones que se encuadran dentro del universo de las necesidades habitacionales y la posibilidad de comprobarlas, medirlas, ponderarlas, todo a los fines de fundamentar una decisión. Y en este proceso inevitablemente construyen recortes que, al crear las categorías que definen quiénes son los sujetos destinatarios de las viviendas, incluye a algunos y excluye a otros. En términos de Fonseca y Cardarello: "cuando determinadas categorías avanzan, otras son designadas para quedar atrás" (2005: 36).

\section{REFERENCIAS BIBLIOGRÁFICAS}

ARGENTINA. MINISTERIO DE PLANIFICACIÓN FEDERAL, INVERSIÓN Y PLANIFICACIÓN FEDERAL. SUBSECRETARÍA DE DESARROLLO URBANO Y VIVIENDA. Presentación Programa Federal de Construcción de viviendas (2004, Julio). Información subprograma de Urbanización de Villas y Asentamientos. Información programa Federal de Construcción de Viviendas "Techo Digno". Documentos disponibles en www.vivienda.gob.ar a noviembre 2013.

BORDIEU, P. (1993). “Espacio social y poder simbólico” en Cosas Dichas. Gedisa Editorial. (1994). El sentido práctico. Editorial Taurus. Madrid. (2009). Las trampas de la cultura. Editorial Paidós.

CARMAN, M. (2011). Las trampas de la naturaleza. Medio ambiente y segregación en Buenos Aires.

CLACSO. Fondo de Cultura Económica.

COLECTIVO SIMBIOSIS CULTURAL. (2011). Vecinocracia (re) tomando la ciudad. Publicación del taller hacer ciudad. Tinta limón ediciones y Editorial Retazos.

20 Bajo las diferentes modalidades de lucha que pueda asumir: toma de tierras, piquete, acampe en la puerta de la casa del Intendente, etc. 
FONSECA, C. \& CARDARELLO, A. (2005). Derechos de los más y menos humanos. En, Tiscornia, S. y Pita, M. V. (Edit.) Derechos humanos, tribunales y policías en Argentina y Brasil. Estudios de Antropología Jurídica. Buenos Aires. Editorial Antropofagia.

GIDDENS, A. (1994). Consecuencias de la modernidad. Editorial Alianza. Madrid.

LEKERMAN, V. (2010). Formas de habitar en una villa de emergencia: redes de relaciones y prácticas de urbanización. En, Revista de Antropología y Ciencias Sociales Kula, Antropólogos del Atlántico Sur, 2. (pp. 65-75).

MANZANO, V. y MORENO, L. (2011). Censar, demandar y acordar. Demandas colectivas y políticas estatales en el Gran Buenos Aires.

OSZLAK, O. (1991) Merecer la Ciudad. Los pobres y el derecho al espacio urbano. Editorial Humanitas-CEDES.

Información Subsecretaría de Desarrollo Urbano y Vivienda, Ministerio de Planificación Federal, inversión pública y servicios: www.vivienda.gob.ar

TALAL A. (2008) ¿Dónde están los márgenes del Estado? En, Cuadernos de Antropología Social, 27. Facultad de Filosofía y Letras. Universidad de Buenos Aires. (pp. 53-62).

TILLY, C. (2000). Acción colectiva. Apuntes de investigación del CECyP.

Trabalho enviado em 04 de agosto de 2014.

Aceito em 05 de dezembro de 2014. 